\title{
Robust Nonlinear State Feedback for Power Systems under Structured Uncertainty
}

\author{
M. A. Mahmud*, M. J. Hossain*, H. R. Pota* \\ * The University of New South Wales at the Australian Defence Force \\ Academy, Northcott Drive, Canberra, ACT 2600, Australia (e-mail: \\ Md.Mahmud@student.adfa.edu.au,m.hossain@adfa.edu.au, and \\ H.Pota@adfa.edu.au).
}

\begin{abstract}
This paper presents a robust nonlinear state feedback linearization approach for power systems with uncertainties. The uncertainties considered here are a class of bounded perturbations to the state model of power systems. The control law is applied through the excitation system of the synchronous generators connected to the power systems to enhance the stability of the system. In this paper, uncertainties are considered for single machine infinite bus (SMIB) system. A Lyapunov-based concept is used to guarantee uniform ultimate boundedness.
\end{abstract}

Keywords: Feedback linearization, Lyapunov function, power systems, stability, uncertainty,.

\section{INTRODUCTION}

For a control scheme to be effective, some details of the system must be available. The details of any system can best be described by the mathematical model. A lot of difficulties may arise while developing a meaningful and realistic mathematical model. The mismatch between the mathematical model and and the true system may lead to serious stability problems for the system, especially when the system is nonlinear; see: Kravaris and Palanki (1988b). Therefore, the design of robust control strategies that consider the model uncertainties are of importance for the design of efficient control systems for nonlinear systems.

In the past decades, one of the most important contributions in field of control theory and application is the development of linear controller design for linear and nonlinear systems with the presence of structured and unstructured uncertainties; see Chao et al. (1994); Lu and Doyle (1997); Liang and Liaw (2003); Shaiju et al. (2007); Petersen (2005). In the design of robust controller for nonlinear system, the uncertainties are considered with the understanding that the error is introduced by linear approximation; see Hossain et al. (2010b,a). In this condition, the key issue is the size of the linear approximation error. In case of small and mild nonlinearities, the uncertainties introduced by the linear approximation is small enough so that it can be rejected by the robust linear controller without too much sacrifice in performance. But in case of highly nonlinear system, the uncertainties are very large as the frequency domain bounds corresponding to linear approximation error are very loose. This leads to the poor performance of the closed-loop system.

Power systems are large, complex, and highly nonlinear systems with constantly varying loads. Control of modern electric power systems becomes more and more challenging as the present trend of power system engineers is to operate the power systems closer to their stability lim- its. Linear control techniques to enhance the stability of the single machine infinite bus (SMIB) systems as well as multimachine power systems are proposed in various papers; see Kundur (1994); Ramos et al. (2005); Dysko et al. (2010); Gurrala and Sen (2010); Gibbard and Vowels (2004); Mahmud et al. (2010a). These linear controllers are designed based on linearized models, which provide satisfactory operation over a small range of operating points and cannot provide stable operation under wide variation of operating regions, i.e., disturbances or uncertainties. Some linear controllers for power systems are designed by considering uncertainties; see Hossain et al. (2009); Yuan and Fang (2009). These controllers provide enhanced stability of the system under uncertainties but still there is some restriction of operating regions.

It is well known that application of nonlinear control theory can provide better operation with respect to the variation of operating points by canceling the inherent system nonlinearities. Feedback linearization control schemes are widely used in power system to design controllers. It deals with the techniques for transforming original system model into equivalent model with a simpler form. The main idea of this approach is to algebraically transform nonlinear system dynamics into a (fully or partly) linear one, so that the linear control techniques can be applied. The performance of a feedback linearizing controller for a single machine infinite bus system as well as for multimachine systems is investigated in; see Mahmud et al. (2010b); Lu et al. (2001). Often the system uncertainties are not considered in the design of the nonlinear controllers proposed in; see Mahmud et al. (2010b); Lu et al. (2001) Another simple form of feedback linearization called direct feedback linearization is proposed in Wang et al. (1995); Guo et al. (2001, 2000), to design controller for single machine infinite bus system as well as for multimachine power systems. In the design of these controllers, the system uncertainties are considered as parametric uncertainties. 


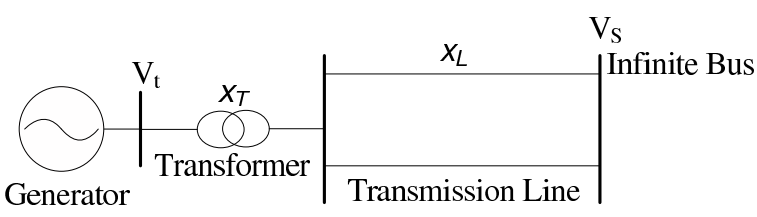

Fig. 1. Power System Model

The aim of this paper is to design a robust nonlinear state feedback law for SMIB System that guarantees stability and performance for all perturbations within the given upper bounds on the modeling error of nonlinear power system using Lyapunov functions. Here, the uncertainties do not need to be parametric as described in Wang et al. (1995); Guo et al. (2001, 2000) but must be representable as a class of perturbations to the state model of power systems to which state-dependent upper bounds are available. This paper considers the case of structured model uncertainty and which is different from including unmodeled higher order dynamics after linearization.

The rest of the paper is organized as follows. In Section 2 , the mathematical modeling of a single machine infinite bus system is given. A brief overview of nonlinear state feedback controller design for power SMIB system is given in Section 3. The uncertainty modeling to design a robust nonlinear state feedback control law is presented in Section 4. In Section 5, a robust nonlinear state feedback control law for SMIB system is proposed. Finally, the paper is concluded with future trends and further recommendation in Section 6.

\section{POWER SYSTEM MODEL}

Power system can be modeled at several different levels of complexities, depending on the intended application of the model. Fig. 1 shows a single machine infinite bus system which is the focus of this paper. Since SMIB system qualitatively exhibits the important aspects of the behavior of a multimachine system and is relatively simple to study, it is extremely useful in studying general concepts of power system stability; see Guo et al. (2001).

With some typical assumptions, the classical third-order dynamical model of a SMIB power system as shown in Fig. 1 can be modeled by the following set of differential equations; see: Kundur (1994); Lu et al. (2001):

Generator mechanical dynamics:

$$
\begin{aligned}
\dot{\delta} & =\omega \\
\dot{\omega} & =-\frac{D}{2 H} \omega+\frac{1}{2 H}\left(P_{m}-P_{e}\right)
\end{aligned}
$$

where $\delta$ is the power angle of the generator, $\omega$ is the rotor speed with respect to synchronous reference, $H$ is the inertia constant of the generator, $P_{m}$ is the mechanical input power to the generator which is assumed to be constant, $D$ is the damping constant of the generator, and $P_{e}$ is the active electrical power delivered by the generator.

Generator electrical dynamics:

$$
\dot{E}_{q}^{\prime}=\frac{1}{T_{d o}}\left(E_{f}-E_{q}\right)
$$

where $E_{q}^{\prime}$ is the quadrature-axis transient voltage of the generator, $E_{q}$ is the quadrature-axis voltage of the generator, $T_{d o}$ is the direct-axis open-circuit transient time constant of the generator, and $E_{f}$ is the equivalent voltage in the excitation coil.

Electrical equations:

$$
\begin{aligned}
E_{q} & =\frac{x_{d \Sigma}}{x_{d \Sigma}^{\prime}} E_{q}^{\prime}-\left(x_{d}-x_{d}^{\prime}\right) \frac{V_{s}}{x_{d \Sigma}^{\prime}} \cos \delta \\
I_{q} & =\frac{V_{s}}{x_{d \Sigma}^{\prime}} \sin \delta \\
P_{e} & =\frac{V_{s} E_{q}^{\prime}}{x_{d \Sigma}^{\prime}} \sin \delta \\
Q_{e} & =\frac{V_{s} E_{q}^{\prime}}{x_{d \Sigma}^{\prime}} \cos \delta-\frac{V_{s}^{2}}{x_{d \Sigma}} \\
V_{t} & =\sqrt{\left(E_{q}^{\prime}-X_{d}^{\prime} I_{d}\right)^{2}+\left(X_{d}^{\prime} I_{q}\right)^{2}}
\end{aligned}
$$

where $x_{d \Sigma}=x_{d}+x_{T}+x_{L}, x_{d \Sigma}^{\prime}=x_{d}^{\prime}+x_{T}+x_{L}, x_{d}$ is the direct-axis synchronous reactance, $x_{d}^{\prime}$ is the direct axis transient reactance, $x_{T}$ is the reactance of the transformer, $x_{L}$ is the reactance of the transmission line, $I_{d}$ and $I_{q}$ are direct and quadrature axis currents of the generator respectively, $V_{s}$ is the infinite bus voltage, $Q_{e}$ is the generator reactive power delivered to the infinite bus, and $V_{t}$ is the terminal voltage of the generator.

Using the electrical equations into the mechanical and electrical dynamics equation (1)-(3) of the system, the complete mathematical model of SMIB system can be written as follows:

$$
\begin{aligned}
\dot{\delta} & =\omega \\
\dot{\omega} & =-\frac{D}{2 H} \omega+\frac{1}{2 H} P_{m}-\frac{1}{2 H} \frac{V_{s} E_{q}^{\prime}}{x_{d \Sigma}^{\prime}} \sin \delta \\
\dot{E}_{q}^{\prime} & =-\frac{1}{T_{d}^{\prime}} E_{q}^{\prime}+\frac{1}{T_{d o}} \frac{x_{d}-x_{d}^{\prime}}{x_{d \Sigma}^{\prime}} V_{s} \cos \delta+\frac{1}{T_{d o}} E_{f}
\end{aligned}
$$

where $T_{d}^{\prime}=\frac{x_{d \Sigma}^{\prime}}{x_{d \Sigma}} T_{d o}$ is the time constant of the field winding. The numerical values of the system parameters are given in Appendix A.

\section{OVERVIEW OF NONLINEAR CONTROLLER DESIGN FOR SMIB SYSTEM}

Let, the nonlinear system can be written by equations of the form

$$
\begin{aligned}
& \dot{x}=f(x)+g(x) u \\
& y=h(x)
\end{aligned}
$$

where $x \in R^{n}$ is the state vector; $u \in R$ is the control vector; $y \in R$ is the output vector; $f(x)$ and $g(x)$ are the $n$-dimensional vector fields in the state space; $h(x)$ is the scalar function of $x$.

The power system model is represented by the equation (4)-(6). These equations have the form of equation (7) where,

$$
x=\left[\begin{array}{lll}
E_{q}^{\prime} & \omega & \delta
\end{array}\right]^{T}
$$




$$
f(x)=\left[\begin{array}{c}
-\frac{1}{T_{d}^{\prime}} E_{q}^{\prime}+\frac{1}{T_{d o}} \frac{x_{d}-x_{d}^{\prime}}{x_{d \Sigma}^{\prime}} V_{s} \cos \delta \\
-\frac{D}{2 H} \omega+\frac{1}{2 H} P_{m}-\frac{1}{2 H} \frac{V_{s} E_{q}^{\prime}}{x_{d \Sigma}^{\prime}} \sin \delta \\
\omega
\end{array}\right]
$$

and

$$
u=E_{f}
$$

The feedback linearizing control input for feedback linearizable systems which satisfy conditions mentioned in Mahmud et al. (2010b); Lu et al. (2001) can be written as Kravaris and Palanki (1988b):

$$
u=\frac{-\sum_{k=0}^{r} \beta_{k} L_{f}^{k}(h)+v}{(-1)^{r-1} \beta_{r}\left\langle d h, a d f_{f}^{r-1}(g)\right\rangle}
$$

where $v$ is the control input of the feedback linearizable systems, $\beta_{k}$ and $\beta_{r}$ are arbitrarily selected numbers, $\mathrm{r}$ is the relative degree of the systems, $L_{f} h(x)$ is the Lie derivative of $h(x)$ along $f(x), a d_{f} g(x)$ is the Lie bracket of $g(x)$ along $f(x)$, and $\langle.,$.$\rangle denotes the inner product in$ $\mathbf{R}^{n}$ i.e.

$$
\langle d h, f\rangle=\frac{\partial h}{\partial x_{1}} f_{1}+\ldots+\frac{\partial h}{\partial x_{n}} f_{n}
$$

For the SMIB system as mentioned by equation (4)-(6), the relative degree, $r$, equals to 2 which can easily be determined by using Lie derivative when the relative speed of the generator $\omega$. The details are shown in the Section 5 of this paper. If we assume, $\beta_{0}=\beta_{1}=\beta_{2}=1$, then we can write, see: Mahmud et al. (2010b); Lu et al. (2001)

$$
\begin{aligned}
\sum_{k=0}^{r} \beta_{k} L_{f}^{k}(h) & =\omega+\left(1-\frac{D}{2 H}\right) \dot{\omega}-\frac{1}{2 H} \frac{V_{s}}{x_{d \Sigma}^{\prime}} \sin \delta \dot{E}_{q}^{\prime} \\
& -\frac{V_{s} E_{q}^{\prime}}{2 H x_{d \Sigma}^{\prime}} \omega^{2} \cos \delta \\
(-1)^{r-1}\left\langle d h, a d f_{f}^{r-1}\right\rangle & =-\frac{V_{s}}{2 H x_{d \Sigma}^{\prime} T_{d o}} \sin \delta
\end{aligned}
$$

and by using the concept of optimal control theory; see Khargonekar et al. (1991), the control input for feedback linearized system can be written as

$$
v=-\Delta \delta-2.29 \Delta \omega-2.14 \Delta \dot{\omega}
$$

By using equation (10)-(12) into equation (9), the fully control input for SMIB system can be obtained. This control input does not include any uncertainties in the system. The modeling of uncertainties and its application to the SMIB system is the key features of the following Sections.

\section{UNCERTAINTY MODELING}

In this section, we will consider the problem of designing a robust controller so that the SMIB systems output $y(t)$ will track a given set point $y_{s p}$; see: Kravaris and Palanki (1988b). By introducing appropriate deviation in the variables, we can rewrite our system so that $y$ represents the tracking error. Here, we would like to design a robustness correction

$$
v=\boldsymbol{R}(x)
$$

so that the system represented by equation (7)-(8) with state feedback equations (9) and (13), has the tracking error that is uniformly ultimately bounded in the sense as described in Kravaris and Palanki (1988b,a). By considering some suitable limitations on the modeling errors

\section{$\Delta f, \quad \Delta g, \quad$ and $\quad \Delta h$}

the tracking error can be bounded uniformly and ultimately. The limitations are given based on the following assumptions; see: Kravaris and Palanki (1988b,a):

Assumption 1. Functions $f, g$, and $h$ are smooth. $\Delta f \in \Sigma_{f}$, $\Delta g \in \Sigma_{g}, \Delta h \in \Sigma_{h}$ where $\Sigma_{f}, \Sigma_{g}, \Sigma_{h}$ are specified sets. This assumption reflects the mathematical completeness of the system.

Assumption 2. The origin $x=0$ is a uniformly asymptotically stable equilibrium point of the unforced nominal system

$$
\dot{x}=f(x)+g(x) u .
$$

In particular there exists a scalar function $V: R^{n} \rightarrow R^{+}$ which is the Lyapunov function and continuous strictly increasing functions $\gamma_{i}: R^{+} \rightarrow R^{+}, i=1,2,3$ satisfying

$$
\begin{aligned}
\gamma_{i}(0) & =0, \quad i=1,2,3 \\
\lim _{r \rightarrow \infty} \gamma_{i}(r) & =\infty, \quad i=1,2
\end{aligned}
$$

such that

$$
\begin{array}{r}
\gamma_{1}(\|x\|) \leq V(x) \leq \gamma_{2}(\|x\|) \\
\left\langle d V, f-\frac{\sum_{k=0}^{r} \beta_{k} L_{f}^{k}(h) g}{(-1)^{r-1} \beta_{r}\left\langle d h, a d f_{f}^{r-1}(g)\right\rangle}\right\rangle \leq \gamma_{3}(\|x\|)
\end{array}
$$

This assumption specifies that the nominal system must be internally stable in the sense that there exist a Lyapunov function. This is a natural assumption because we cannot expect robustness unless the nominal system is internally stable.

Assumption 3. Function $h(x)$ vanishes at the origin $x=0$ which represents the tracking error.

Assumption 4. For each $\Delta f \in \Sigma_{f}$ and $\Delta g \in \Sigma_{g}$ there exist mappings $\Delta f^{*}: R^{n} \rightarrow R$ and $\Delta g^{*}: R^{n} \rightarrow R$ satisfying

$$
(-1)^{r-1} \beta_{r}\left\langle d h, a d f_{f}^{r-1}(g)\right\rangle \Delta f=g \Delta f^{*}, \quad \Delta g=g \Delta g^{*}(15)
$$

for all $x \in R^{n}$. This assumption defines the matching of the theory. The structure of the uncertainty is also defined by this assumption.

Assumption 5. There exist a scalar function $\mu: R^{n} \rightarrow R$ such that

$$
1+\Delta g^{*} \geq \mu(x)>0
$$

for every $\Delta g \in \Sigma_{g}$. Moreover, $\mu(x)$ is bounded: 


$$
\mu^{0} \geq \mu(x)>0 \quad \forall x \in R^{n} .
$$

Assumption 6. There exist a scalar function $\phi: R^{n} \rightarrow R$ such that

$$
\phi(x) \geq \frac{1}{\mu(x)}\left|\Delta f^{*}-\left[\sum_{r=0}^{k} \beta_{k} L_{f}^{k}(h)\right] \Delta g^{*}\right|
$$

for every $\Delta f \in \Sigma_{f}$ and $\Delta g \in \Sigma_{g}$.

Assumption 7. There exists $\delta_{h}>0$ such that

$$
|\Delta h| \leq \delta_{h}
$$

for every $\Delta h \in \Sigma_{h}$.

Assumptions (5)-(7) define the state dependent uncertainty band for $\Delta f, \Delta g$, and $\Delta h$. Now, if the assumptions (1)-(7) are consistent, by considering the uncertainties, i.e., the modeling error the nonlinear system can be written as

$$
\begin{aligned}
& \dot{x}=[f(x)+\Delta f]+[g(x)+\Delta g] u, \quad x(0)=x_{0} \\
& y=h(x)+\Delta h
\end{aligned}
$$

where $\Delta f \in \Sigma_{f}, \Delta g \in \Sigma_{g}, \Delta h \in \Sigma_{h}$, and the state feedback

$$
u=\frac{-\sum_{k=0}^{r} \beta_{k} L_{f}^{k}(h)+v}{(-1)^{r-1} \beta_{r}\left\langle d h, a d f_{f}^{r-1}(g)\right\rangle}
$$

where

$$
v=-\phi(x) \eta_{\epsilon} \frac{\mu^{0} \phi(x)\langle d V, g\rangle(x)}{(-1)^{r-1} \beta_{r}\left\langle d h, a d f_{f}^{r-1}(g)\right\rangle} .
$$

Here, $\eta_{\epsilon}$ is the saturation function

$$
\eta_{\epsilon}= \begin{cases}\frac{\zeta}{\epsilon} & \text { if }|\zeta| \leq \epsilon \\ \frac{\zeta}{|\zeta|} & \text { if }|\zeta|>\epsilon\end{cases}
$$

with

$$
\epsilon<\lim _{r \rightarrow \infty} \gamma_{3}(r)
$$

Equation (22) is a continuous approximation of discontinuous control law

$$
v=-\phi(x) \operatorname{sgn} \frac{\mu^{0} \phi(x)\langle d V, g\rangle(x)}{(-1)^{r-1} \beta_{r}\left\langle d h, a d f_{f}^{r-1}(g)\right\rangle}
$$

as $\epsilon \rightarrow 0$. Due to the discontinuity in the above mentioned control law cannot be implemented in practical situation. The tracking error will also be uniformly ultimately bounded if equation (22) is replaced by unsaturated control law, see: Kravaris and Palanki (1988a)

$$
v=-\frac{\mu^{0}[\phi(x)]^{2}}{\epsilon} \frac{\langle d V, g\rangle(x)}{(-1)^{r-1} \beta_{r}\left\langle d h, a d f_{f}^{r-1}(g)\right\rangle}
$$

From equation (24), it is observed that the uncertainty band $\phi(x)$ is feeding back with a nonlinear gain. This theory also indicates that the value of $\epsilon$ should be as small as possible. However, a very small value of $\epsilon$ may excite the unmodeled higher order dynamics, which are not taken into account in the theory. Practically, we need to choose $\epsilon$ by simulation.

\section{ROBUST NONLINEAR STATE FEEDBACK CONTROL FOR POWER SYSTEM}

When a large sudden fault occurs on power systems, the reactance of the transmission line, $x_{L}$ changes a lot. This results in the changes in line parameters to the system uncertainty. The power system model with the uncertainty can be written as

$$
\begin{aligned}
\dot{\delta} & =\omega \\
\dot{\omega} & =-\frac{D}{2 H} \omega+\frac{1}{2 H} P_{m}-\frac{1}{2 H} \frac{V_{s} E_{q}^{\prime}}{x_{d \Sigma}^{\prime}} \sin \delta+\zeta_{P_{e}} \\
\dot{E}_{q}^{\prime} & =-\left(\frac{1}{T_{d}^{\prime}}+\Delta T_{d}^{\prime}\right) E_{q}^{\prime}+\frac{1}{T_{d o}} \frac{x_{d}-x_{d}^{\prime}}{x_{d \Sigma}^{\prime}} V_{s} \cos \delta+\zeta_{I_{d}} \\
& +\left(\frac{1}{T_{d o}}+\Delta T_{d o}\right) E_{f}
\end{aligned}
$$

where $\zeta_{P_{e}}$ and $\zeta_{I_{d}}$ is the uncertainty in the system that occurs. As the change in line parameters causes uncertainty in delivered active power and line flow and that is why the consideration of $\zeta_{P_{e}}$ and $\zeta_{I_{d}}$ as uncertainty is meaningful. Since the uncertainty in the line reactance also causes uncertainty in $T_{d o}$ and $T_{d}^{\prime}$, so $\Delta T_{d o}$ and $\Delta T_{d}^{\prime}$ are also chosen as uncertainty. Now we need to select the upper bound of this uncertainty.

The above power system model equation (25)-(27) can be written as

$$
\begin{aligned}
& \dot{x}=[f(x)+\Delta f]+[g(x)+\Delta g] u, \quad x(0)=x_{0} \\
& y=h(x)+\Delta h
\end{aligned}
$$

where the modeled system is

$$
\begin{aligned}
& \dot{x}=f(x)+g(x) u \\
& y=h(x) .
\end{aligned}
$$

We can write

$$
\begin{gathered}
x=\left[\begin{array}{lll}
E_{q}^{\prime} \omega & \delta
\end{array}\right]^{T} \\
f(x)=\left[\begin{array}{c}
-\frac{1}{T_{d}^{\prime}} E_{q}^{\prime}+\frac{1}{T_{d o}} \frac{x_{d}-x_{d}^{\prime}}{x_{d \Sigma}^{\prime}} V_{s} \cos \delta \\
-\frac{D}{2 H} \omega+\frac{1}{2 H} P_{m}-\frac{1}{2 H} \frac{V_{s} E_{q}^{\prime}}{x_{d \Sigma}^{\prime}} \sin \delta \\
\omega
\end{array}\right] \\
g(x)=\left[\begin{array}{ccc}
\frac{1}{T_{d o}} & 0 & 0
\end{array}\right]^{T} \\
\Delta f=\left[\begin{array}{c}
\Delta T_{d}^{\prime} E_{q}^{\prime}+\zeta_{I_{d}} \\
\zeta_{P_{e}} \\
0
\end{array}\right] \\
\Delta g=\left[\begin{array}{ccc}
\Delta T_{d o} & 0
\end{array}\right]^{T}
\end{gathered}
$$

Now the aim is to design a robust state feedback controller based on the modeled SMIB system. This can be done through the following steps:

Step 1. Calculation of relative order The Lie derivative of the function $L_{f}^{r-1} h(x)$ along the 
vector field $g(x)$ is not equal to zero in a neighborhood $\Omega$, i.e.

$$
L_{g} L_{f}^{r-1} h(x) \neq 0
$$

then the system is said to have relative degree $r$ in $\Omega$. For SMIB system, this can be calculated as follows:

We consider

$$
y=h(x)=\omega
$$

Therefore,

$$
L_{g} L_{f}^{1-1} h(x)=L_{g} h(x)=\frac{\partial h(x)}{\partial x} g(x)=0
$$

Now before calculating $L_{g} L_{f}^{2-1} h(x)$, first we need to calculate

$$
L_{f}^{2-1} h(x)=L_{f} h(x)=\frac{\partial h(x)}{\partial x} f(x)=\dot{\omega}
$$

which leads to the following result

$$
L_{g} L_{f}^{2-1} h(x)=L_{g} L_{f} h(x)=\frac{\partial\left(L_{f} h(x)\right)}{\partial x} g(x) \neq 0
$$

Therefore, the relative degree of the system is two.

Step 2. Calculation of feedback control law From Section 3, the feedback control law can be written as

$$
u=\frac{v-\frac{1}{2 H T_{d o}} \frac{V_{s} E_{q}}{x_{d \Sigma}^{\prime}} \sin \delta-\frac{D}{2 H} \dot{\omega}-\frac{V_{s} E_{q}^{\prime}}{2 H x_{d \Sigma}^{\prime}} \omega \cos \delta}{-\frac{V_{s}}{2 H x_{d \Sigma}^{\prime} T_{d o}} \sin \delta}
$$

Step 3. Calculation of Lyapunov function for unforced system

The unforced system can be obtained by putting $v=0$. Using Krasovskii's method, see: Slotine and Li (1991) and using all the numerical values of SMIB system parameters, the Lyapunov function can be written as

$$
\begin{aligned}
V & =\omega^{2}+0.2517{E_{q}^{\prime}}^{2}-0.3580 E_{q}^{\prime} \cos \delta+0.1273 \cos ^{2} \delta \\
& +0.1273+0.0977{E_{q}^{\prime}}^{2}+0.0094{E_{q}^{\prime}}^{2} \sin ^{2} \delta-0.0352 \omega \\
& -0.0109 \omega \sin \delta-0.0606 \omega^{2} \sin \delta
\end{aligned}
$$

Step 4. Calculation of $\Delta f^{*}$ and $\Delta g^{*}$ Equation (15) To determine $\Delta f^{*}$ and $\Delta g^{*}$,first we need to bound the amount of uncertainty. In the normal operating condition of the system, the initial value, $\delta_{0}=72^{\circ}$. During the faulted condition, if one of the transmission line disconnected, i.e., if there is $50 \%$ change in the line reactance, then the upper bound of the uncertainty is given as

$\Delta T_{d}^{\prime} \leq 2.42, \quad \zeta_{I_{d}} \leq 0.09, \quad \Delta T_{d o} \leq 0.5, \quad$ and $\quad \zeta_{P_{e}} \leq 0.5$ Therefore, by using equation (15), we can write

$$
\begin{aligned}
& \Delta f^{*}=-0.06 \sin \delta\left(E_{q}^{\prime}+0.14\right) \\
& \Delta g^{*}=0.072
\end{aligned}
$$

Step 5. Calculation of $\mu(x), \mu^{0}$ (Equation 16 and 17) Using Assumption 5 we can set $\mu(x)$ as any value between

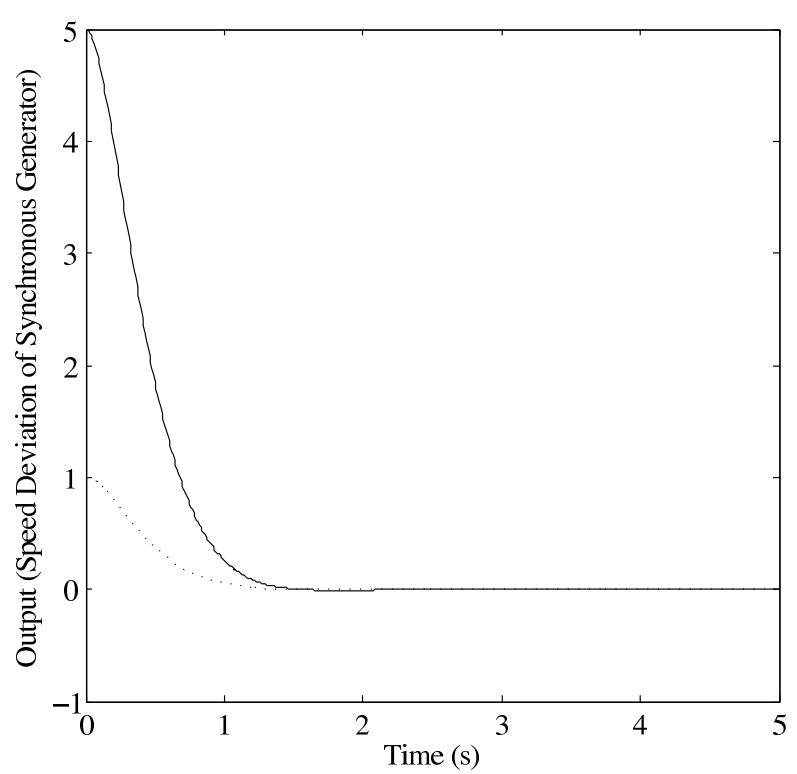

Fig. 2. Uniform Ultimate Boundedness of Tracking Error (Dashed line represents for initial value of $\delta_{0}=72^{\circ}$ and solid line for $\delta_{0}=58^{\circ}$ )

$0<\mu(x) \leq 1.072$. For this application we set $\mu(x)=\mu^{0}=$ 0.2 .

Step 6. Calculation of $\phi(x)$ (Equation 18)

Using equation $(18), \phi(x)$ can be written as

$$
\begin{aligned}
\phi(x) & =\frac{1}{0.2} \mid-0.06 \sin \delta\left(E_{q}^{\prime}+0.14\right)-\left(0.014 E_{q} \sin \delta-0.3125 \dot{\omega}\right. \\
& \left.-0.014 E_{q}^{\prime} \omega \cos \delta\right) 0.072 \mid
\end{aligned}
$$

Step 7. Calculation of $\langle d V, g\rangle$ We can calculate $\langle d V, g\rangle$ as

$$
\langle d V, g\rangle=\frac{\partial V}{\partial E_{q}^{\prime}} g_{1}+\frac{\partial V}{\partial \omega} g_{2}+\frac{\partial V}{\partial \delta} g_{3}
$$

Therefore,

$$
\langle d V, g\rangle=\frac{1}{6.9}\left(0.69884 E_{q}^{\prime}-0.3560 \cos \delta+0.0188 E_{q}^{\prime} \sin ^{2} \delta\right)
$$

By putting all the values into equation (24), the robust control law is obtained. Using this control law, the unform ultimate boundedness of the tracking error, i.e., the speed deviation of the generator can be described by Fig. 2 .

In Fig. 2, the dashed line shows the boundedness of the output when the initial value, $\delta_{0}=72^{\circ}$ and the solid line shows that of $\delta_{0}=58^{\circ}$. During these simulations the values of $\epsilon$ is taken as 0.003 .

\section{CONCLUSION}

In this paper, a robust nonlinear state feedback controller is designed for a given upper bound in the modeling error of a SMIB system. The performance of the controller is simulated under two different initial values of the system. The controller guarantees the desired tracking error when the uncertainties are representable as a class of perturbations to the state model of power systems. Future works 
will deal with the extension of the proposed method to handle power systems models with dynamic loads as well as for the power systems with unmodeled higher order dynamics.

\section{Appendix A. POWER SYSTEM PARAMETERS}

The parameters used for the SMIB system are given below: Synchronous generator parameters:

$x_{d}=1.863 \mathrm{pu}, x_{d}^{\prime}=0.257 \mathrm{pu}, H=8 \mathrm{~s}, T_{d o}=6.9 \mathrm{~s}$ $D=5, \omega_{0}=314.159$.

Transformer Parameter: $x_{T}=0.127 p u$.

Transmission Line Parameters: $x_{L}=0.4853 \mathrm{pu}$.

Infinite bus voltage, $V_{s}=1.00 \mathrm{pu}$.

Mechanical Power Input, $P_{m}=0.9 \mathrm{pu}$.

\section{REFERENCES}

Chao, A., Athans, M., and Stein, G. (1994). Stability robustness to unstructured uncertainties for nonlinear systems under feedback linearization. In Proc. of the 33rd Conference on Decission and Control.

Dysko, A., Leithead, W.E., and O'Reilly, J. (2010). Enhanched power system stability by coordinated PSS design. IEEE Trans. on Power Systems, 25(1), 413-422.

Gibbard, M.J. and Vowels, D.J. (2004). Reconcilation of methods of compensation for psss in multimachine systems. IEEE Trans. on Power Systems, 19(1), 10421051.

Guo, G., Hill, D.J., and Wang, Y. (2000). Nonlinear output stabilization control for multimachine power systems. IEEE Tans. on Circuit and Systems- Part 1, 47(1), 4652.

Guo, G., Hill, D.J., and Wang, Y. (2001). Global transient stability and voltage regulation for power systems. IEEE Trans. on Power Systems, 16(4), 678-688.

Gurrala, G. and Sen, I. (2010). Power system stabilizers design for interconnected power systems. IEEE Trans. on Power Systems, 25(2), 1042-1051.

Hossain, M.J., Pota, H.R., Ugrinovoski, V., and Ramos, R.A. (2009). Excitation control for large disturbances in power systems with dynamic loads. In Proc. of 2009 IEEE PES General Meeting.

Hossain, M.J., Pota, H.R., Ugrinovoski, V., and Ramos, R.A. (2010a). Decentralised robust STATCOM control for wind farms to augment dynamic transfer capability. Journal of Renewable and Sustainable Energy, 2(2), 022701(1)-022701(20).

Hossain, M.J., Pota, H.R., Ugrinovoski, V., and Ramos, R.A. (2010b). Simultaneous STATCOM and pitch angle control for improved fault-ride capabilty of fixed speed wind turbines. IEEE Trans. on Sustainable Energy, 1(3), In Press.

Khargonekar, P.P., Petersen, I.P., and Zhou, K. (1991). Robust stabilization of uncertain linear systems: Quadratic stabilizability and $H^{\infty}$ control theory. IEEE Trans. on Automatic Control, AC-35, 356-361.

Kravaris, C. and Palanki, S. (1988a). A lyapunov approach for robust nonlinear state feedback synthesis. IEEE Trans. on Automatic Control, AC-33, 1188-1191.

Kravaris, C. and Palanki, S. (1988b). Robust nonlinear state feedback under structured uncertainty. AIChE Journal, 34(7), 1119-1127.
Kundur, P. (1994). Power System Stability and Control. McGraw-Hill, New York.

Liang, Y.W. and Liaw, D.C. (2003). Robust control of nonlinear affine systems. Applied Mathematics and Computation, 137, 337-347.

Lu, Q., Sun, Y., and Mei, S. (2001). Nonlinear Control Systems and Power System Dynamics. Kluwer Academic Publishers, Boston/Dordrecht/London.

Lu, W.M. and Doyle, J.C. (1997). An approach to quantify the technical benefits of distributed generation. IEEE Trans. on Automatic Control, 42(12), 1654-1662.

Mahmud, M.A., Hossain, M.J., and Pota, H.R. (2010a). Effect of large dynamic loads on interconnected power systems with power oscillation damping controller (PODC). Accepted for Publication in the 20th Australasian Universities Power Engineering Conference.

Mahmud, M.A., Hossain, M.J., and Pota, H.R. (2010b). Nonlinear excitation control of power systems with dynamic loads via feedback linearization. Accepted for Publication in the 20th Australasian Universities Power Engineering Conference.

Petersen, I.R. (2005). A notion of controllability for uncertain linear systems with structured uncertainty. In Proc. of the 44th Conference on Decission and Control.

Ramos, R.A., Martins, A.C.P., and Bretas, N.G. (2005). An improved methodolgy for the design of power system damping controllers. IEEE Trans. on Power Systems, 20(4), 1938-1945.

Shaiju, A., Petersen, I., and James, M. (2007). Guaranteed cost LQG control of uncertain linear stochastic quantum systems. In Proc. of the 2007 American Control Conference.

Slotine, J.J.E. and Li, W. (1991). Applied Nonlinear Control. Prentice-Hall, New Jersey.

Wang, Y., Hill, D.J., and Guo, G. (1995). Nonlinear decentralized control for multimachine power system transient stability enhancement. In Proc. of Int. Symp. on Electric Power Engineering, 435-440.

Yuan, S.Q. and Fang, D.Z. (2009). Robust PSS parameters design using a trajectory sensitivity approach. IEEE Trans. on Power Systems, 24(2), 1042-1051. 